\title{
Optimized Live Volumetric Imaging with Light Sheet Microscopy and Related Strategies
}

\author{
Thai V. Truong ${ }^{1}$ \\ 1. Translational Imaging Center, University of Southern California, Los Angeles, USA.
}

Biological imaging often involves a balancing of tradeoffs, to maximize the collection of desired information while working under the constraints of physical principles and of the biological sample. In optical imaging, this balancing act can be described as the tradeoffs between the parameters of resolution, imaging speed, and penetration depth, Fig. 1A, where high performance in one parameter necessarily requires sacrifices in the other two, subjected to the constraint of photo-damage, defined as encompassing both photo-bleaching of the fluorophores and biological photo-induced toxicity.

In recent years, light sheet microscopy, aka Selective Plane Illumination Microscopy (SPIM), has emerged as an imaging modality that, while still operating under the tradeoffs described above, opens up a new parameters space where simultaneously higher performance in all of the parameters of resolution, speed, depth can be achieved, compared with conventional imaging modalities [1]. In SPIM, Fig. 1B, the sample is illuminated from the side and signal contrast is detected in the orthogonal direction with a wide-field camera. The unique side illumination restricts the optical excitation to only the plane where useful information is being collected, leading to reduced background, enhanced SNR, and reduced photo-damage, and thus allows for better performance compared with conventional modalities. In this contribution I will describe three on-going, unpublished projects in our lab where we build upon the basic SPIM technology to further develop and optimize novel imaging strategies, to meet the needs of a diverse set of live volumetric imaging applications.

The high performance of SPIM across the parameters space of speed, depth, and resolution makes it a unique choice for neuroscience, especially in the emerging applications of imaging live, intact brains of model organisms such as C. Elegans, Drosophila, and zebrafish. In these animals, taking advantage of their brains' small size and optical accessibility, the latest innovations in imaging technology are starting to enable imaging of up to the whole, live brains, with the aim to record and understand neuronal structures and functions across scales, from cellular to whole-brain, and correlating them with the animals' behavior. I will describe our efforts where we use SPIM to carry out structural and functional imaging of the live zebrafish larval brain, to observe the synaptic structure and neuronal functional activity pattern in the context of learning, memory, and sleep/wake homeostasis. Our imaging yields large and information-rich datasets that present a challenge in the processing and analysis, yet hold the promise of providing new insights into understanding neuronal processes at the brain-wide level.

Developmental biology is another field where SPIM has been making significant contributions. Our lab is interested in understanding the embryonic development of the heart, particularly in the roles that biophysical forces generated by the heart beating and blood flow play in the cardiac developmental program. Toward that end, we want to image the beating heart in its full 3D details at high enough spatio-temporal resolution to follow subcellular structures throughout the beating cycle. Experimentally this translates to the requirements of imaging with at least $\sim 1 \mu \mathrm{m}$ resolution, covering a volume of $200 \mu \mathrm{m} \times 150 \mu \mathrm{m} \times 150 \mu \mathrm{m}$ (axial depth), at speed $>50$ volumes/s, which is simply out of reach for conventional imaging approaches. Taking the advantage of the periodic nature of the heart beating, we 
develop an imaging approach, termed Macroscopic Phase Stamping (MaPS), which allows us to use retrospective gating to reconstruct the dynamic 3D beating heart. In this method, we employ an additional imaging path to obtain a low-resolution macroscopic bright-field image of the beating heart, captured synchronously with the optically-sectioned high-resolution fluorescence SPIM images, so that for every fluorescence image we have a corresponding bright-field image that contains the macroscopic spatio-temporal information about the cardiac phase. We then use the bright-field images to quantitatively determine the phase of each fluorescence image, which then enables assembling the images into the correct sequence in both phase and axial depth.

For the general case of fast dynamic biological processes that are not periodic, retrospective gating approaches such as MaPS will not work, hence there is a need for a synchronous volumetric imaging approach, where a single snapshot could capture spatial information over an extended volume. Such capability has been demonstrated with Light Field Microscopy (LFM), where the 3D light field coming from the sample space is recorded in a single 2D image, capturing information from the 3D volume extended above and below the native focal plane, albeit at reduced spatial resolution due to the diffraction limit. Computational reconstruction is used to generate the 3D images of the sample from the 2D light field images. Up until now, LFM has been implemented with wide-field illumination, illuminating the entirety of the sample, even though only a part of the sample is meaningfully captured in the reconstruction. Thus, wide-field illumination produces extraneous background and noise, degrading the contrast of the final reconstructed images. To address this problem, taking a lesson from the selective plane illumination strategy of SPIM, we implement Selective Volume Illumination to LFM, illuminating only the volume of interest, thus significantly reducing the background and providing higher contrast to the final reconstructed image. With this approach, we are able to achieve synchronous volumetric imaging rates of up to 100 volumes/s, at cellular resolution, with tissue-scale volume coverage - a performance that we believe will enable many previously untenable applications [2].

\section{References:}

[1] For reviews and key publications, see the "Light-sheet Microscopy Collection", Nature Methods, http://www.nature.com/nmeth/collections/lightsheetmicroscopy/index.html

[2] The author acknowledges funding from the Moore Foundation (GBMF3396), NIH

(1R01MH107238-01), and NSF (007289-00001).

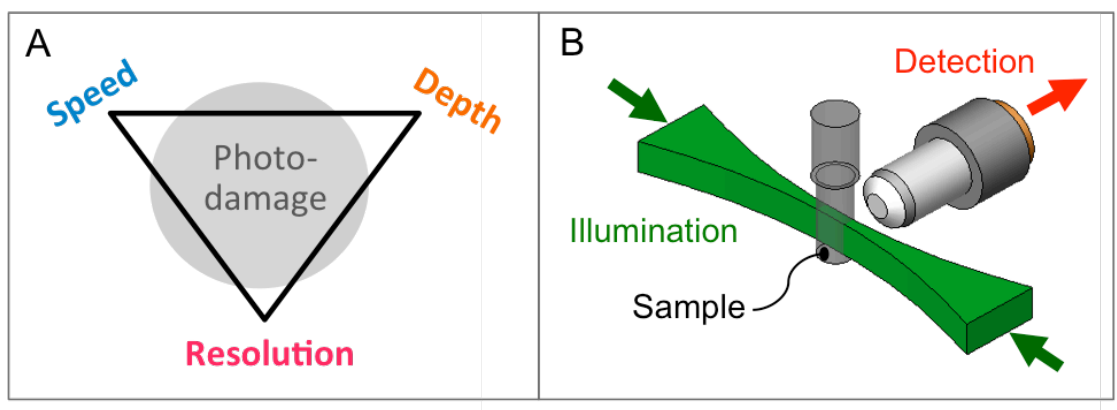

Figure 1. (A) Optical imaging performance tradeoffs in imaging speed, penetration depth into 3dimensional samples, and resolution, all under the constraint minimizing photo-damage. (B) Basic principles of light sheet microscopy, where a nominal sheet of light illuminates the sample from the side, and the signal contrast is detected in the orthogonal direction with a camera. 